ARTICLE

Received 26 Feb 2013 | Accepted 12 Jul 2013 | Published 7 Aug $2013 \quad$ DOI: 10.1038/ncomms3297

\title{
Disk-cylinder and disk-sphere nanoparticles via a block copolymer blend solution construction
}

Jiahua Zhu', Shiyi Zhang ${ }^{2,3}$, Ke Zhang ${ }^{4}$, Xiaojun Wang ${ }^{5}$, Jimmy W. Mays ${ }^{5}$, Karen L. Wooley ${ }^{2}$ \& Darrin J. Pochan ${ }^{6}$

Researchers strive to produce nanoparticles with complexity in composition and structure. Although traditional spherical, cylindrical and membranous, or planar, nanostructures are ubiquitous, scientists seek more complicated geometries for potential functionality. Here we report the simple solution construction of multigeometry nanoparticles, disk-sphere and diskcylinder, through a straightforward, molecular-level, blending strategy with binary mixtures of block copolymers. The multigeometry nanoparticles contain disk geometry in the core with either spherical patches along the disk periphery in the case of disk-sphere particles or cylindrical edges and handles in the case of the disk-cylinder particles. The portions of different geometry in the same nanoparticles contain different core block chemistry, thus also defining multicompartments in the nanoparticles. Although the block copolymers chosen for the blends are important for the definition of the final hybrid particles, the control of the kinetic pathway of assembly is critical for successful multigeometry particle construction.

\footnotetext{
${ }^{1}$ Center of Nanophase Materials Sciences, Oak Ridge National Laboratory, Oak ridge, Tennessee 37831, USA. ${ }^{2}$ Departments of Chemistry and Chemical Engineering, Texas A\&M University, College Station, Texas 77843, USA. ${ }^{3}$ Department of Chemistry, Washington University in St Louis, St Louis, Missouri 63130, USA. ${ }^{4}$ Department of Chemistry and Chemical Biology, Northeastern University, Boston, Massachusetts 02115, USA. ${ }^{5}$ Department of Chemistry, University of Tennessee, Knoxville, Tennessee 37996, USA. ${ }^{6}$ Department of Materials Science and Engineering, University of Delaware, Newark, Delaware 19716, USA. Correspondence and requests for materials should be addressed to K.L.W. (email: wooley@chem.tamu.edu) or to D.J.P. (email: pochan@udel.edu).
} 
C ompositional and geometrical complexity of nano-sized objects has long been an important topic in materials science leading to novel properties and applications in fields such as nano-medicine ${ }^{1}$, organic photonics ${ }^{2}$ and electronics ${ }^{3}$. Beyond the considerable success in confining multiple materials and compositions into nano-objects with simple geometric shapes such as spheres, cylinders and vesicles ${ }^{4-7}$, recent attention has focused on the impact of particle geometry on optical ${ }^{8}$, electronic ${ }^{9}$ and biomedical ${ }^{10}$ properties. However, a systematic understanding of nanoparticle construction with both compositional and geometrical complexity does not yet exist.

Nano-objects have been successfully constructed via solution supramolecular assembly from a wide range of multi-component molecular building units, such as block copolymers ${ }^{5-7}$, block copolypeptides $^{11}$ and $\mathrm{DNA}^{8}$, with well-defined structures and functions manipulated by constituent molecular architecture and component chemistry. Block copolymers consist of two or more chemically different polymer components linked together by covalent bonding. Block copolymer molecules form specific geometries of solution-assembled, micelle-like nanoparticles determined by the relative volume of the respective blocks, the interaction between polymer block segments and the interaction of the respective blocks with the solvent (Fig. 1a,b) ${ }^{12}$. In a solvent that selectively solubilizes one block of a block copolymer, molecules aggregate predominately into bilayer membranes (that is, vesicles), cylinders or spheres. Nanostructure geometry can be controlled precisely by changing the relative block lengths, by

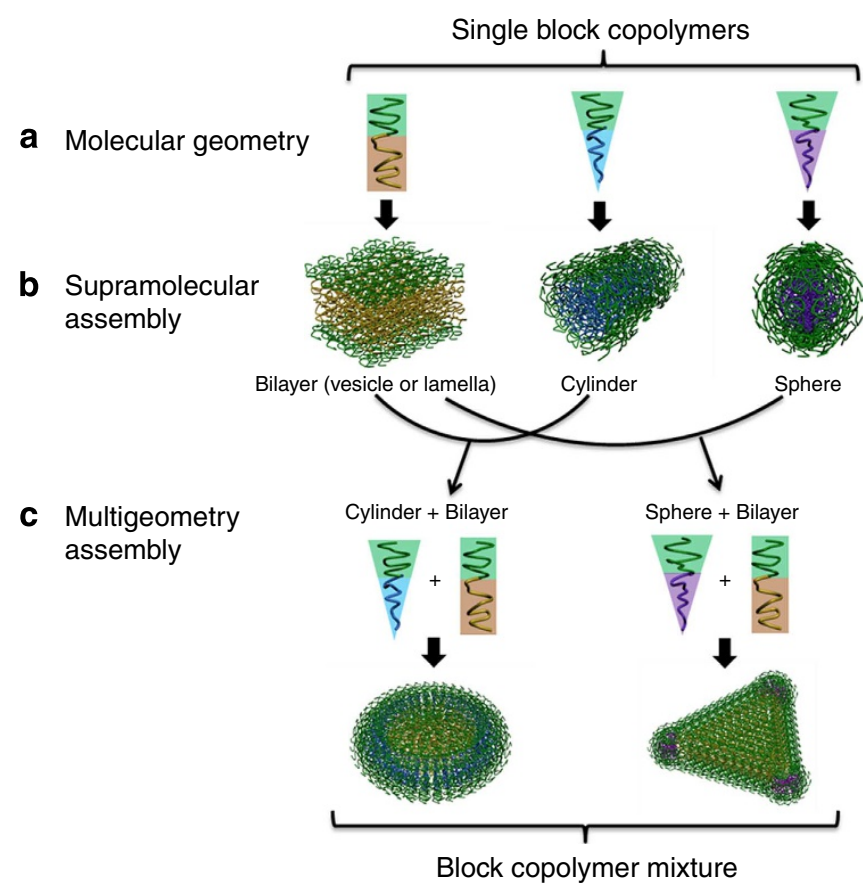

Figure 1 | Schematic design for multigeometry nanoparticle construction.

Single block copolymers possess (a) specific molecular geometries based on relative length of block and solvophilicity/solvophobicity of the respective blocks. The individual block copolymers can assemble into (b) bilayer membranes (for example, vesicles), cylinders or spheres, through desired solution assembly. By taking advantage of molecular design and synthesis, varied block copolymers are assembled into diverse geometries at the same assembly condition. Herein, (c) multigeometry nanoparticles are achievable from co-assembly of selected mixtures of block copolymers in unlike molecular geometries, for example, cylinderforming and bilayer-forming block copolymer mixture, or sphere-forming and bilayer-forming block copolymer mixture. adding external stimuli such as solvent mixing ${ }^{5}$ or addition of other molecules that specifically associate with and tune a desired block volume in an assembly 13,14, and by changing the solvent processing kinetic pathway ${ }^{15,16}$. Owing to the slow molecular exchange dynamics between block copolymer assemblies and the solvent that prevents global thermodynamic equilibration ${ }^{17}$, nanoparticles with kinetically trapped, complex nanostructures are achievable.

The ability to kinetically trap block copolymers into nanoparticles and observe local equilibration within the particles provides an excellent opportunity to construct complex, multi-component nano-objects for multifunctional, hierarchical materials $^{18,19}$. In single diblock copolymer solution suspensions, assemblies with locally mixed geometries, for example, cylinders with sphere end-caps and Y-junctions between cylinders, have been found at relative block compositions that lie between molecular compositions that form traditional spheres and cylinders or cylinders and vesicles ${ }^{20}$. In blends of different block copolymers with identical block chemistry but different block lengths, cylinders with sphere end-caps and vesicles with cylinder branches have been observed due to the local trapping and segregation of block copolymers with different molecular weights ${ }^{21}$. Alternately, one can purposely mix different block copolymers with unlike block chemistries into multi-component nanoparticles with proper kinetic processing. The limited examples of this strategy have focused on block copolymer molecules with similar molecular weights and relative block fractions for the production of blended spheres ${ }^{22,23}$, cylinders that contain multiple block copolymers ${ }^{15,23-25}$ and blended vesicles $^{26,27}$.

Here, we develop a straightforward solution processing strategy for multigeometry nanoparticle construction that produces hybrid geometry, disk-sphere or disk-cylinder polymeric nanoparticles. Final multi-geometry nanoparticles are part disk and either part cylinder or part sphere and are formed simply through blending of two unlike block copolymers into mixed nanoparticles that subsequently segregate locally into sub-domains of distinct geometry and distinct core block chemistry. We show that this strategy can be used to construct novel soft matter nanostructures with designed compositions and geometries from simple block copolymers.

\section{Results}

Multigeometry discoid assembly strategy. In this present work, nanoparticles with hybrid discoid morphologies have been constructed with mixed block copolymers assembled through kinetic control in solution. Rather than closing into vesicles, disks ${ }^{28,29}$ are flat bilayer sheets that are restricted to finite size. Due to inherent geometrical anisotropy, discoid structures exhibit notable shear field-responsive capabilities for prolonged discoid particle blood circulation time $\mathrm{s}^{30}$, are used for membrane protein structure determination via ${ }^{1} \mathrm{H}$ NMR spectroscopy ${ }^{31}$, are used as templates for drug delivery ${ }^{32}$ and for porous material construction ${ }^{33}$. The difficulty in bilayer disk assembly is that the rim around the edge of a discrete disk is a high energy defect; to avoid the exposure of hydrophobic block to the aqueous medium, the molecules around the edge may rearrange to adopt a much higher curvature, as opposed to the flat interfacial curvature that the molecules favour throughout the interior of the nanoparticle ${ }^{34}$ (Supplementary Fig. S1). An approach to form disk structures is to cut vesicles into disks through blending with other block copolymer molecules that prefer to adopt a higher interfacial curvature after assembly in order to stabilize a disk edge. One successful small molecule example of this blending strategy is the formation of discoid bicelles through the mixing of long-chain 
phospholipids that form planar bilayers surrounded by shortchain lipids on the edge that shield the long-chain lipid-tails from water ${ }^{35}$. With proper design of block components and lengths, macromolecular block copolymers exhibit diverse morphologies at the same solution condition, that is, vesicle, cylinder or sphere, where molecules express increasing interfacial curvature, respectively (Fig. 1). Therefore, new, multigeometry disk nanoparticles were achievable from solution co-assembly of a bilayerforming block copolymer and a cylinder-forming or sphereforming block copolymer, respectively.

Disk-cylinder hybrid nanoparticles. Both molecules of the designed binary block copolymer blends presented here possessed the same poly(acrylic acid) (PAA) hydrophilic block chemistry with similar block lengths but possessed different, incompatible hydrophobic blocks, that is, polystyrene (PS) or polyisoprene (PI). Figure 2 displays the circular, disk-like nanoparticles assembled through a defined tetrahydrofuran (THF) and water solventmixing pathway (vide infra) from an equal molar blend of $\mathrm{PAA}_{118}-b-\mathrm{PI}_{176}$ (the subscripts refer to the number-averaged degrees of polymerization) and $\mathrm{PAA}_{99}-b-\mathrm{PS}_{125}$ diblock copolymers in the presence of small, di-functional organoamines, $2,2^{\prime}$-(ethylenedioxy)diethylamine (EDDA). Mixed with the same amount of EDDA diamines at an amine-to-acid ratio of 0.5 , neat $\mathrm{PAA}_{118^{-}}-b-\mathrm{PI}_{176}$ and neat $\mathrm{PAA}_{99}-b-\mathrm{PS}_{125}$ assembled into vesicles (Fig. 2a) and cylindrical micelles (Fig. 2c), respectively, at high water content $\left(20 \mathrm{v} \%\right.$ THF, $\left.80 \mathrm{v} \% \mathrm{H}_{2} \mathrm{O}\right)$. The thickness of the vesicular membrane and cylinder diameter as measured from an average of 100 particles in cryo-TEM were $35 \pm 3 \mathrm{~nm}$ and $33 \pm 2 \mathrm{~nm}$, respectively. Upon co-assembly of the two diblock copolymers with EDDA at the same amine-to-acid ratio of 0.5 , circular particles with diameter $\leq 100 \mathrm{~nm}$ were formed with homogeneous contrast in cryo-TEM imaging, Fig. 2d, indicative of the formation of flat, disk-like nanostructures. The co-existing oblate and needle-like structures result from disk particles at different orientations with respect to the electron beam. The morphology was further confirmed by $\mathrm{OsO}_{4}$ staining in cast films of the nanoparticles collected from the same sample solution (Fig. 2e, $\mathrm{OsO}_{4}$ selectively stained $\mathrm{PI}$ by reacting with carbon double bonds). A dark, central, flat region is where the PAA- $b$-PI molecules were observed in each particle, surrounded by a grey rim area rich in PAA- $b$-PS. A minor population of larger particles (size $>100 \mathrm{~nm}$, Fig. 2f) was observed with a grey cylindrical PAA- $b$-PS tail extending out of the disk. The width of the disk rim was constant, approximately half of the diameter of the PAA$b$-PS cylinder tails, suggesting a half cylinder of PAA-b-PS molecules packing around PAA- $b$-PI bilayer as illustrated in Fig. 2g,h. Additional cryo-TEM and cast-film data of disk-cylinder hybrid particles are shown in Supplementary Figs S3,S4, respectively.

The morphology of each disk-cylinder hybrid particle was defined by the materials trapped inside. As shown in Fig. 3, small particles in diameter of $\sim 50$ to $80 \mathrm{~nm}$ possessed a round discoid membrane of PAA- $b$-PB and a half cylinder rim of PAA- $b$-PS. As particle size increased, the PAA- $b-\mathrm{PB}$ membrane generally elongated into oblate disk for an increased rim area to pack more PAA- $b$-PS half cylinders around the edge. With even more PAA- $b$-PS trapped in a particle, full cylinders would form and extend out from the oblate disks. By simply comparing the relative areas of dark (PI-containing block copolymer) to light (PS-containing block copolymer) in the hybrid particle
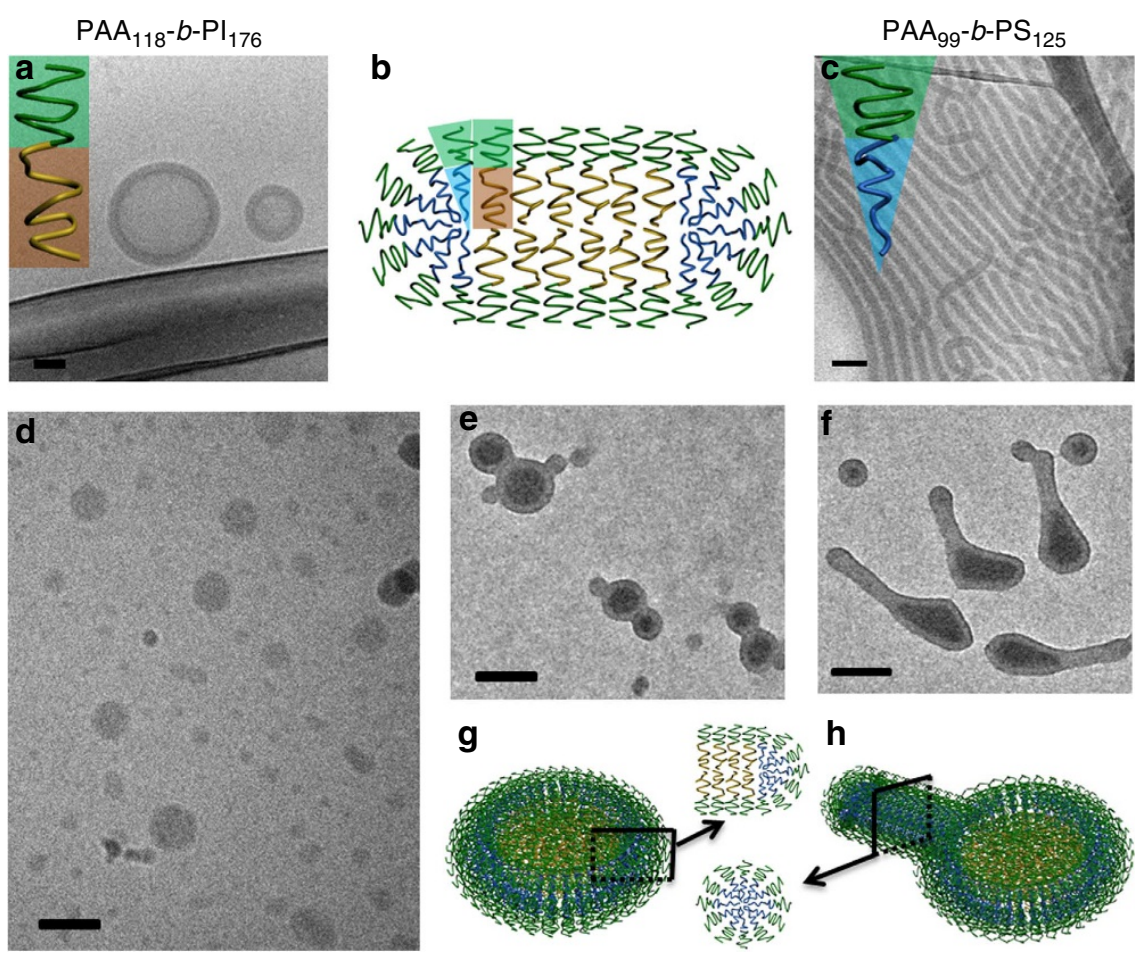

Figure 2 | Disk-cylinder multigeometry nanoparticles. Block copolymer(s) were assembled in a THF and water solvent mixture (20 v\% $\mathrm{THF}, 80$ v\% $\mathrm{H}_{2} \mathrm{O}$ ) in the presence of EDDA at an amine-to-acid ratio $=0.5$. (a) Cryo-TEM image of vesicles formed by $\mathrm{PAA}_{118}$-b-PI $\mathrm{I}_{176}$. (b) Cross-sectional schematic shows the desired organization of block copolymer molecules into disk assemblies. (c) Cryo-TEM image of cylindrical micelles formed by PAA Pg- $_{9}-\mathrm{PS}_{125}$.

(d) Cryo-TEM image and (e,f). OsO $\mathrm{O}_{4}$ stained TEM images of hybrid particles formed by equal molar blend of $\mathrm{PAA}_{118}-\mathrm{b}-\mathrm{Pl}_{176}$ and $\mathrm{PAA} \mathrm{A}_{99}-b$-PS $125 ;(\mathbf{f})$ shows the formation of the minority component tailed-disk structure. Proposed distributions of block copolymer molecules in the disk and tailed-disk assemblies are illustrated in $(\mathbf{g}, \mathbf{h})$. EDDA molecules that closely associate with the PAA blocks within the assembled nanoparticles are not shown in the schematics for clarity. Scale bar, $100 \mathrm{~nm}$. 


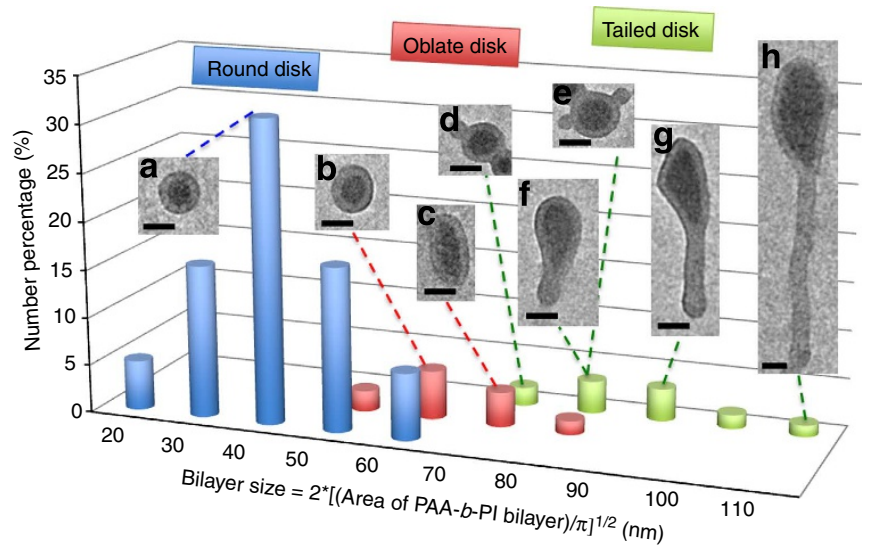

Figure 3 | Statistical evaluation of disk-cylinder hybrid morphologies.

Column graph shows the number percentage of disk-cylinder

multigeometry nanoparticles of slightly different morphology (produced from analysis of 100 particles in $\mathrm{OsO}_{4}$ stained TEM image). Blue, red and green columns are corresponding to populations of round disk, oblate disk and tailed-disk, respectively. The sizes of the bilayer section of the particles were calculated from the area of dark PAA-b-PI disk region in the particles by an equation of bilayer size $=2^{\star}[(\text { Area of PAA- } b \text {-PI bilayer }) / \pi]^{1 / 2}$. The bilayer size is equal to the diameter of the central dark region of PAA-b-PI assuming a round disk section to simply give a measure of size comparison between particle disk sections. Insert images are $\mathrm{OsO}_{4}$ stained TEM showed the selected particle morphologies in the same sample of round disk (a), oblate disks $(\mathbf{b}, \mathbf{c})$ and tailed disks $(\mathbf{d}-\mathbf{h})$ of different bilayer sizes indicated by dash lines. Scale bar, $50 \mathrm{~nm}$.

population, the volume ratio of PS to PI in the particles overall well represent the equimolar ratio of the two block copolymers in the initial blend of PAA- $b$-PS and PAA- $b$-PI.

The success in multigeometry assembly from block copolymer mixtures relies on two phenomena: proper nanoparticle confinement and subsequent internal organization. Kinetic control of the assembly process through the proper solvent-mixing pathway is critical for success with proper nanoparticle confinement. The solvent-mixing pathway began with dissolution of a desired amount of block copolymers mixed with EDDA diamines in THF. THF is a good solvent for the block copolymers, while the EDDA complexes with the PAA blocks to form cores of inverse micellelike aggregate structures. Subsequent addition of water had the combined effect of aggregating the hydrophobic blocks into new nanoparticle hydrophobic cores, while concurrently swelling PAA-EDDA complexed hydrophilic blocks into the new nanoparticle shells. Final micelle-like nanoparticles were obtained when water addition reached a volume ratio of 4:1 water to THF. The complexation of the EDDA amines with PAA acid groups impacted the final morphology in two critical aspects. First, the amines help to tune the shell volumes of the final assembly and thus define the geometry of nanoparticle that a block copolymer would like to adopt ${ }^{26}$. Second, the initial complexation of the PAA and amines in pure THF forces the different block copolymers to reside in the same nanoparticles during assembly, consequently producing particles with mixed hydrophobic cores at higher water content that undergo local, internal phase separation to form multigeometry particles. Obviously, the assembly conditions (solvent composition, block molecular weight, amount and type of amine) must provide for dynamic behaviour within the formed nanoparticles so that local phase separation of unlike hydrophobic blocks can produce the final, multigeometry nanoparticles. Block copolymer blends in an absence of diamines assembled into two separate, ill-defined nanoparticle phases of the two respective diblock copolymers due to the lack of initial confinement in the same nanoparticle in neat THF (Supplementary Fig. S2). In addition, assembly with excessive addition of diamines (amine-toacid ratio $>1.0$ ) produced only spherical nanoparticles for both block copolymers at the desired solution mixture. Therefore, we narrowed the studied amine-to-acid ratio to between 0.3 and 1.0 in order to find appropriate conditions for desired, different particle geometries to be blended into the same nanoparticle with kinetic control. An additional critical aspect of the multigeometry particle assembly strategy is that the presence of the amine-acid complexation and the large molecular weight of the block copolymers both serve to significantly slow chain exchange with the solvent ${ }^{17}$. All of these effects, together, explain why block copolymer chains phase segregate and equilibrate locally within the same nanoparticle. This intraparticle segregation results in the highly interesting and unique expression of the respective block copolymer preferred bilayered or cylindrical geometries within the same nanoparticle.

Disk-sphere hybrid nanoparticles. Through this kinetically controlled assembly strategy, sphere-forming block copolymer was also forced to assemble with a vesicle-forming block copolymer and form disk-sphere hybrid nanoparticles. Under the same solution assembly conditions as the vesicle-cylinder blend vide supra, a vesicle-forming triblock copolymer $\mathrm{PAA}_{150}$-blockpoly(methyl acrylate $)_{60}$-block- $\mathrm{PS}_{240}\left(\mathrm{PAA}_{150}-b-\mathrm{PMA}_{60}-b-\mathrm{PS}_{240}\right)$ and a sphere-forming diblock $\mathrm{PAA}_{75}$-block-polybutadiene 104 $\left(\mathrm{PAA}_{75}-b-\mathrm{PB}_{104}\right)$ were blended at a $4: 1$ molar ratio to produce disk-sphere multigeometry nanoparticles as shown in Fig. 4 and Supplementary Fig. S5. The triblock and diblock copolymers possessed the same PAA block chemistry but different hydrophobic blocks. A large difference from the earlier disk-cylinder blend is that the lengths of the respective hydrophilic or hydrophobic blocks of the two block copolymers were substantially different. The thickness of the vesicular membranes and sphere diameter as measured from an average of 100 particles in cryoTEM were $54 \pm 5 \mathrm{~nm}$ and $32 \pm 5 \mathrm{~nm}$, respectively, in the samples of the individual block copolymers (Fig. 4a,c). In the $\mathrm{OsO}_{4}$ stained cryo-TEM images (Fig. $4 \mathrm{~d}, \mathrm{OsO}_{4}$ selectively stained $\mathrm{PB}$ by reacting with double bonds), disk nanoparticles with a broad range of sizes $(50-300 \mathrm{~nm})$ and number of hemispherical caps (1-6, Fig. 4e-j) were obtained simultaneously. The hybrid particle formation was achieved owing to combined contributions from both PAA- $b$-PB and PAA- $b$-PMA- $b$-PS to stabilize the rim area: sphere-forming PAA- $b$-PB aggregated into hemi-spheres capping the edge apices of the disks, whereas the remaining edge boundaries between the hemispherical caps consisting of PAA- $b$ PMA- $b$-PS tended to be straight, thus limiting the length of higher energy disk edge defect. The original blend ratio is qualitatively observed in the more dominant population of small particles. In larger particles, there is much more disk formation in the hybrid disk-sphere particles. There appears to be a maximum number of sphere caps per disk edge distance that is stable in the larger hybrid particles. Therefore, the excess PAA- $b$-PB must be aggregated into spherical particles that have broken away from the hybrid particles (see the many, very small particles in Fig. $4 \mathrm{~d}$ ). The PAA- $b$-PMA- $b$-PS molecules at the disk straight edges between the hemispherical caps adopt frustrated packing at the edge, as illustrated in Fig. 4b. This frustrated packing shields the hydrophobic core of the disk from solvent, as the hybrid particles do not aggregate together, as would be expected if there were exposed hydrophobic edges.

\section{Discussion}

In summary, we used a molecular-level geometric blending strategy for complex block copolymer nanoparticle construction 

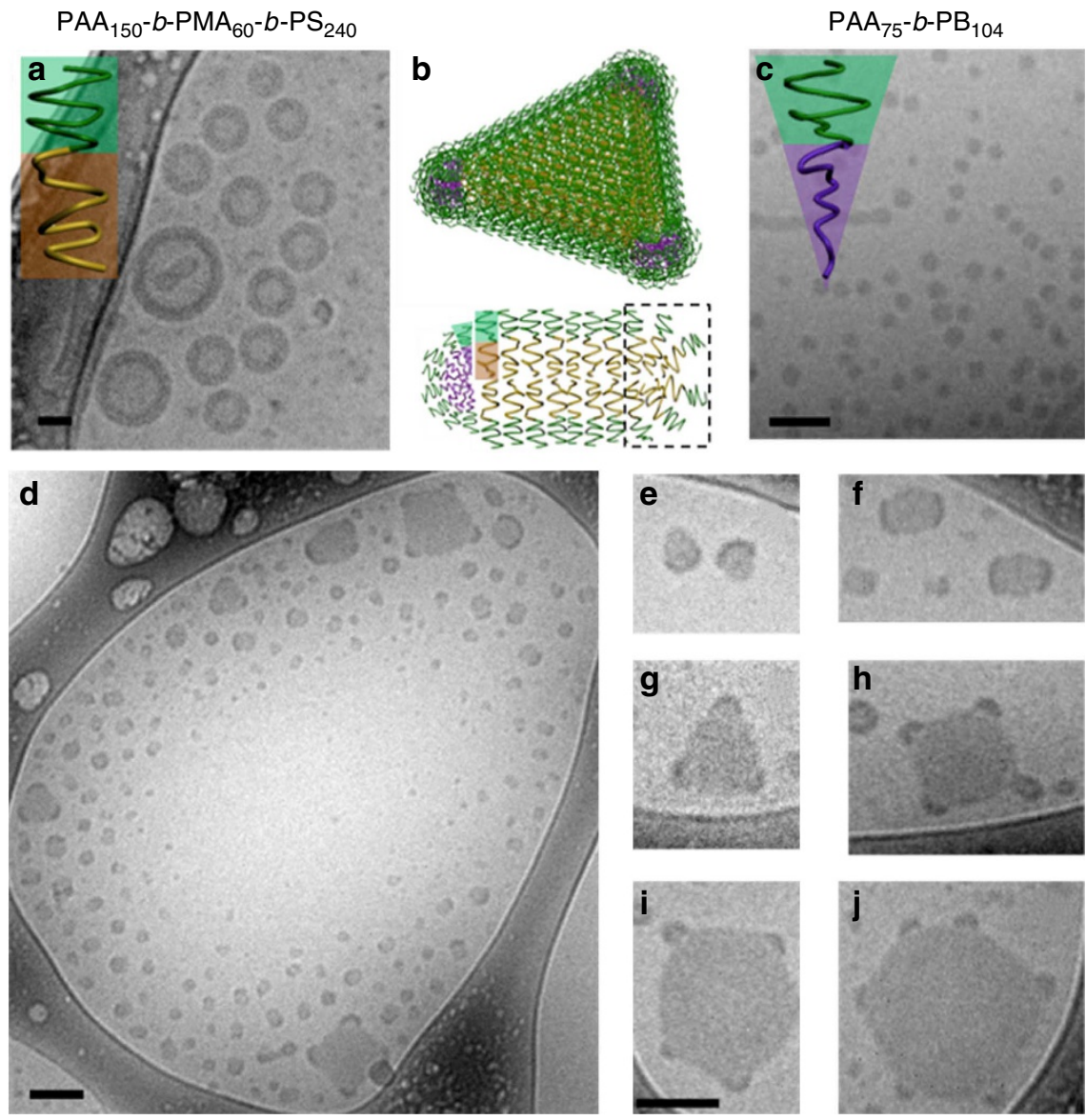

Figure 4 | Disk-sphere multigeometry nanoparticles. Block copolymer(s) were assembled in a THF and water solvent mixture (20 v\% $\left.\mathrm{THF}, 80 \mathrm{v} \% \mathrm{\textrm {H } _ { 2 }} \mathrm{O}\right)$ in the presence of EDDA at an amine-to-acid ratio $=0.5$. (a) Cryo-TEM image of vesicles formed by $\mathrm{PAA}_{150}-b-\mathrm{PMA}_{60}-b-\mathrm{PS}_{240}$. $(\mathbf{c})$. Cryo-TEM image of spherical micelles formed by $\mathrm{PAA}_{75}-b-\mathrm{PB}_{104}$. (d) $\mathrm{OsO}_{4}$ stained cryo-TEM image of hybrid particles formed by 4:1 molar ratio blend of $\mathrm{PAA}_{150}-b$ - $\mathrm{PMA}_{60}-b-$ $\mathrm{PS}_{240}$ and $\mathrm{PAA}_{75}-b-\mathrm{PB}_{104}$. (e-j) OsO $\mathrm{O}_{4}$ stained cryo-TEM images show the selected disk-sphere multigeometry nanoparticles with 1-6 sphere caps on the disk edges. Proposed distributions of block copolymer molecules in the hybrid nanoparticles are illustrated in (b). $\mathrm{PAA}_{150}-b-\mathrm{PMA}_{60}-b-\mathrm{PS}_{240}$ also reside in the disk rim area between spherical edge caps of $\mathrm{PAA}_{75}-b-\mathrm{PB}_{104}$ as marked by the dashed square. PMA and PS hydrophobic blocks are both coloured gold for clarity. EDDA molecules that closely associate with the PAA blocks within the assembled nanoparticles are not shown in the schematics for clarity. Scale bar, $100 \mathrm{~nm}$.

with designed compartments and multiple geometries. Disk-cylinder and disk-sphere multigeometry nanoparticles have been obtained through the simple solvent-mixing process. With further exploration in size and shape, variety of the molecular units and further advancement in kinetic control and solution processing, more well-defined, complex multigeometry nanoparticles are expected from new, binary, ternary or higher order blends with desired relative amount of each component and potential multifunction. In addition to the attractive shapes and complex morphologies that these multigeometry nanostructures possess globally, the local differentiation of composition leads to segregation of physical, chemical and mechanical properties within a single nanoscopic framework. Advanced investigation of the sub-nanoparticle properties and the incorporation of functional sub-units is expected to lead to highly sophisticated materials that may serve as components for future technological device construction.

\section{Methods}

Sample preparation. Block copolymers mixed at certain blending ratio first were dissolved in THF to produce a 0.1 weight per cent homogeneous solution of block copolymer. Next, EDDA was added to give a desired amine-to-acid molar ratio (for example, $0.5: 1.0)$ and to form inverse-like micelle aggregates. Subsequent water addition was performed by slow water titration performed on a syringe pump stage (KD Scientific Syringe Pump, KDS 100). One millilitre Water per hour was added to $0.5 \mathrm{ml}$ THF polymer solution with vigorous stirring. Afterwards, sample solutions were sealed in vials and aged for 1 day before characterization.

Transmission electron microscopy. TEM imaging was performed on a Tecnai G2 12 microscope operating at an accelerating voltage of $120 \mathrm{kV}$. TEM samples were prepared by applying a drop of polymer solution (about $2-4 \mu \mathrm{l}$ ) onto a carboncoated copper TEM grid and allowing the solvents to evaporate under ambient conditions. Images were collected on a Gatan CCD.

Cryogenic transmission electron microscopy. A Gatan cryo-holder system was used to examine the samples in a Tecnai G2 12 microscope at voltage $120 \mathrm{kV}$. Sample preparation was performed in the Vitrobot virtrification system. A droplet of the sample solution $(10 \mu \mathrm{l})$ was dipped on a lacey carbon-coated copper TEM grid. The specimen was blotted and plunged into a liquid ethane container cooled by liquid nitrogen. The vitrified samples were transferred to a Gatan 626 cryo-holder and cryo-transfer stage cooled by liquid nitrogen. During TEM observation, the cryo-holder was maintained below $-170 \mathrm{~K}$ to prevent the sublimation of vitreous water and THF. The digital images were collected by Gatan low-dose CCD.

Staining methods. A vapour staining method was applied to cast-film TEM samples. Dried TEM grids were placed on a $25 \times 75 \times 1 \mathrm{~mm}^{3}$ glass slide to sit in a sealed $100 \mathrm{ml}$ glass bottle suspended above $1 \mathrm{ml} 4 \mathrm{wt} \%$ osmium tetroxide aqueous 


\section{Table 1 | Molecular Characteristics of diblock copolymer used in this study.}

\begin{tabular}{lcccc} 
Sample ID & $\boldsymbol{N}_{\text {PAA }}{ }^{\star}$ & $\boldsymbol{N}_{\text {PS }^{*}}$ & $\boldsymbol{M}_{\mathbf{n}}\left(\times \mathbf{1 0}^{\mathbf{4}} \mathbf{D a}\right)$ & $\mathbf{P D I}\left(\boldsymbol{M}_{\mathbf{w}} / \boldsymbol{M}_{\mathbf{n}}\right)^{\dagger}$ \\
\hline DE010307-11 & 99 & 125 & 2.03 & 1.08
\end{tabular}

PAA, poly(acrylic acid); PDI, polydispersity index; PS, polystyrene.

${ }^{\star} N$ is the degree of polymerization determined by ${ }^{1} \mathrm{H}$ NMR of purified product $P t B A_{9 g}-b-P_{125}$.

$\dashv$ PDI of PtBA $99-b-$ PS $_{125}$, the precursor of PAA-PS, was determined by GPC using polystyrene standards and THF as solvent.

\section{Table 2 | Molecular Characteristics of triblock copolymer used in this study.}

\begin{tabular}{lccccc} 
Sample ID & $\boldsymbol{N}_{\text {PAA }}{ }^{\star}$ & $\boldsymbol{N}_{\text {PMA }^{\star}}$ & $\boldsymbol{N}_{\text {PS }^{\star}}$ & $\mathbf{M}_{\mathbf{n}}\left(\times \mathbf{1 0}^{\mathbf{4}} \mathbf{D a}\right)$ & PDI $\left(\mathbf{M}_{\mathbf{w}} / \mathbf{M}_{\mathbf{n}}\right)^{\dagger}$ \\
\hline SZ-ii-188 & 150 & 60 & 240 & 4.09 & 1.12
\end{tabular}

PAA, poly(acrylic acid); PDI, polydispersity index; PMA, poly (methyl acrylate); PS, polystyrene. ${ }^{\star} N$ is the degree of polymerization determined by ${ }^{1} \mathrm{H}$ NMR of purified product $\mathrm{PtBA}_{150}-b-\mathrm{PMA}_{60}$ $b-\mathrm{PS}_{240}$

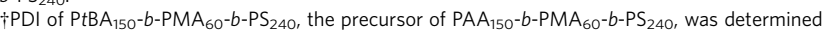
by GPC using polystyrene standards and THF as solvent.

solution (purchased from Electron Microscopy Sciences). $\mathrm{OsO}_{4}$ vapour staining process lasted from $6-24 \mathrm{~h}$ for different samples.

A solution staining method was applied to cryo-TEM samples by directly mixing $20 \mu \mathrm{l} 4 \mathrm{wt} \%$ osmium tetroxide aqueous solution with $1 \mathrm{ml}$ assembly sample solution upon gird preparation and imaging.

Synthesis of PAA-b-PS diblock copolymer. The PAA-b-PS diblock copolymer used in this study were synthesized by atom transfer radical polymerization, following previous reported procedures ${ }^{36}$. Briefly, $t$-butyl acrylate (ca. 200 equiv.) was polymerized by using ethyl 2-bromopropionate ( $c a$. 1.0 equiv.) as initiator, $\mathrm{CuBr}$ (ca. 1.9 equiv.) and $\mathrm{CuBr}_{2}$ (ca. 0.1 equiv.) as catalysts and $N, N, N^{\prime}, N^{\prime}, N^{\prime \prime}-$ pentamethyldiethylenetriamine (PMDETA, $c a$. 2.0 equiv.) as ligand.

Polymerization was performed at $50^{\circ} \mathrm{C}$ and monitored by withdrawing small aliquots for GPC and ${ }^{1} \mathrm{H}$ NMR analyses. $\mathrm{P} \mathrm{BA}_{99}$ was then extended into $\mathrm{P} t \mathrm{BA}_{99}-b$ $\mathrm{PS}_{125}$ by chain extension polymerizing with styrene ( $c a .500$ equiv.), using $\mathrm{CuBr}$ (ca. 3.0 equiv.) and PMDETA (ca. 3.0 equiv.) as catalyst and ligand, at a temperature of $55^{\circ} \mathrm{C}$. The polymerization reactions were quenched at styrene conversion of $25 \%$ by freezing in liquid nitrogen. The $t$-butyl-protected diblock copolymer were then transformed into $\mathrm{PAA}_{99}-b-\mathrm{PS}_{125}$ by reacted with iodotrimethylsilane (ca. 3.0 equiv.) following a reported deprotection protocol ${ }^{14}$ The molecular characteristics of the diblock copolymer is listed in Table 1.

Synthesis of PAA-b-PI diblock copolymers. The precursor PI- $b$-P $t$ BuA for producing well-defined PAA- $b$-PI diblock copolymers used in this study were synthesized by anionic polymerization of isoprene and tert-butyl acrylate employing high vacuum techniques according to standard protocol ${ }^{37,38}$. Tert-Butyl groups of $\mathrm{P} t \mathrm{BA}$ blocks were cleaved by mild hydrolysis as reported previously ${ }^{37}$. PI: $M_{\mathrm{n}}=1.2 \times 10^{4} \mathrm{~g} \mathrm{~mol}^{-1}$ (SEC-LLS), PDI, 1.05 ; PI- $b$-P $t$ BA: $M_{\mathrm{n}}=2.3 \times 10^{4} \mathrm{~g} \mathrm{~mol}^{-1}$ (SEC), PDI, 1.19. Molar ratio isoprene/acrylic acid $=1.5: 1$ by ${ }^{1} \mathrm{H}$ NMR.

Synthesis of PAA-b-PMA-b-PS triblock copolymers. The $\mathrm{PAA}_{150}-b-\mathrm{PMA}_{60}-b$ $\mathrm{PS}_{240}$ used in this study was synthesized by an atom transfer radical polymerization, following previous reported procedure ${ }^{39}$. In brief, $t$-butyl acrylate (ca. 400 equiv.) was polymerized by using ethyl 2-bromopropionate (ca. 1.0 equiv.) as initiator, $\mathrm{CuBr}$ (ca. 1.9 equiv.) and $\mathrm{CuBr}_{2}$ (ca. 0.1 equiv.) as catalysts, and PMDETA (ca. 2.0 equiv.) as ligand. Polymerization was performed at $50^{\circ} \mathrm{C}$ and monitored by withdrawing small aliquots for GPC and ${ }^{1} \mathrm{H}$ NMR analyses. $\mathrm{P} t \mathrm{BA}_{150}$ was then extended into $\mathrm{P} \mathrm{BA}_{150}-b-\mathrm{PMA}_{60}$ by chain extension polymerizing with methyl acrylate (ca. 500 equiv.), using $\mathrm{CuBr}$ (ca. 3.0 equiv.) and PMDETA (ca. 3.0 equiv.) as catalyst and ligand, at a temperature of $55^{\circ} \mathrm{C}$. $\mathrm{P} \mathrm{BA}_{150}-b-\mathrm{PMA}_{60}$ was used as a macroinitiator, to extend into $\mathrm{P}^{\mathrm{BA}} \mathrm{A}_{150}-b-\mathrm{PMA}_{60}-b-\mathrm{PS}_{240}$, by using $\mathrm{CuBr}$ (ca. 3.0 equiv.) as catalysts, and bipyridine (ca. 4.0 equiv.) as ligand, at the temperature of $60^{\circ} \mathrm{C}$. $\mathrm{P} \mathrm{BA}_{150}-b$ - $\mathrm{PMA}_{60}-b$ - $\mathrm{PS}_{240}$ was transformed into $\mathrm{PAA}_{150^{-}} b$ $\mathrm{PMA}_{60}-b-\mathrm{PS}_{240}$ by reaction with iodotrimethylsilane (ca. 3.0 equiv.). The molecular characteristics of the triblock copolymer is listed in Table 2.

PAA-b-PB diblock copolymers. PAA- $b$-PB diblock copolymer was purchased from Polymer Source, Inc. and used as received. Composition and molecular weight were tested by ${ }^{1} \mathrm{H}$ NMR: $\mathrm{PAA}(5450)-b-\mathrm{PB}(5600), \mathrm{PDI}=1.10$ as indicated by the company.
Others. EDDA was purchased from Sigma-Aldrich Corporation. THF was purchased from Acros Organics.

\section{References}

1. Wagner, V., Dullaart, A., Bock, A. K. \& Zweck, A. The emerging nanomedicine landscape. Nat. Biotechnol. 24, 1211-1217 (2006)

2. Huynh, W. U., Dittmer, J. J. \& Alivisato, A. P. Hybrid nanorod-polymer solar cells. Science 295, 2425-2427 (2002).

3. Mirkin, C. A., Letsinger, R. L., Mucic, R. C. \& Storhoff, J. J. A DNA-based method for rationally assembling nanoparticles into macroscopic materials. Nature 382, 607-609 (1996).

4. Caruso, F., Caruso, R. A. \& Möhwald, H. Nanoengineering of inorganic and hybrid hollow spheres by colloidal templating. Science 282, 1111-1114 (1998).

5. Zhang, L. \& Eisenberg, A. Multiple morphologies of 'crew-cut' aggregates of polystyrene- $b$-poly(acrylic acid) block copolymers. Science 268, 1728-1731 (1995).

6. Li, Z. B., Kesselman, E., Talmon, Y., Hillmyer, M. A. \& Lodge, T. P. Multicompartment micelles from ABC miktoarm stars in water. Science 306, 98-101 (2004)

7. Lodge, T. P., Rasdal, A., Li, Z. B. \& Hillmyer, M. A. Simultaneous segregated storage of two agents in a multicompartment micelle. J. Am. Chem. Soc. 127, 17608-17609 (2005).

8. Anton, K. et al. DNA-based self-assembly of chiral plasmonic nanostructures with tailored optical response. Nature 483, 311-314 (2012).

9. Borys, N. J., Walter, M. J., Huang, J., Talapin, D. V. \& Lupton, J. M. The role of particle morphology in interfacial energy transfer in CdSe/CdS heterostructure nanocrystals. Science 330, 1371-1374 (2010).

10. Geng, Y. et al. Shape effects of filaments versus spherical particles in flow and drug delivery. Nat. Nano 2, 249-255 (2007).

11. Holowka, E. P., Sun, V. Z., Kamei, D. T. \& Deming, T. J. Polyarginine segments in block copolypeptides drive both vesicular assembly and intracellular delivery. Nat. Mater. 6, 52-57 (2007).

12. Israelachvili, J. N. Intermolecular and Surface Forces 3rd edn (Academic Press, 2011).

13. Stoykovich, M. P. et al. Directed assembly of block copolymer blends into nonregular device-oriented structures. Science 308, 1442-1446 (2005).

14. Pochan, D. J. et al. Toroidal triblock copolymer assemblies. Science 306, 94-97 (2004).

15. Cui, H., Chen, Z., Zhong, S., Wooley, K. L. \& Pochan, D. P. Block copolymer assembly via kinetic control. Science 317, 647-650 (2007).

16. Gröschel, A. H. et al. Precise hierarchical self-assembly of multicompartment micelles. Nat. Commun. 3, 710 (2012).

17. Choi, S.-H., Lodge, T. P. \& Bates, F. S. Mechanism of molecular exchange in diblock copolymer micelles: hypersensitivity to core chain length. Phys. Rev. Lett. 104, 047802 (2010).

18. Rupar, P. A., Chabanne, L., Winnik, M. A. \& Manners, I. Non-centrosymmetric cylindrical micelles by unidirectional growth. Science 337, 559-562 (2012).

19. Zhu, J., Zhang, S., Zhang, F., Wooley, K. L. \& Pochan, D. J. Hierarchical assembly of complex block copolymer nanoparticles into multicompartment superstructures through tunable interparticle associations. Adv. Funct. Mater. 23, 1767-1773 (2013).

20. Jain, S. \& Bates, F. S. On the origins of morphological complexity in block copolymer surfactants. Science 300, 460-464 (2003).

21. Jain, S. \& Bates, F. S. Consequences of nonergodicity in aqueous binary PEO-PB micellar dispersions. Macromolecules 37, 1511-1523 (2004).

22. Li, G., Shi, L., Ma, R., An, Y. \& Huang, N. Formation of complex micelles with double-responsive channels from self-assembly of two diblock copolymers. Angew. Chem. Int. Ed. 45, 4959-4962 (2006).

23. Pochan, D. J. et al. Multicompartment and multigeometry nanoparticle assembly. Soft Matter 7, 2500-2506 (2011).

24. Li, Z., Hillmyer, M. A. \& Lodge, T. P. Control of structure in multicompartmen micelles by blending $\mathrm{m}-\mathrm{ABC}$ star terpolymers with $\mathrm{AB}$ diblock copolymers. Macromolecules 39, 765-771 (2006).

25. Wang, X. et al. Cylindrical block copolymer micelles and co-micelles of controlled length and architecture. Science 317, 644-647 (2007).

26. Christian, D. A. et al. Spotted vesicles, striped micelles and Janus assemblies induced by ligand binding. Nat. Mater. 8, 843-849 (2009).

27. LoPresti, C. et al. Controlling polymersomes surface topology at the nanoscale by membrane confined polymer/polymer phase separation. ACS Nano 5, 1775-1784 (2011).

28. Edmonds, W. F., Li, Z. B., Hillmyer, M. A. \& Lodge, T. P. Disk micelles from nonionic coil - coil diblock copolymers. Macromolecules 39, 4526-4530 (2006).

29. Li, Z. et al. Disc morphology and discto-cylinder tunability of poly(acrylic acid)-b-poly(methyl acrylate)-b-polystyrene triblock copolymer solution-state assemblies. Langmuir 21, 7533-7539 (2005). 
30. Merkel, T. J. et al. Using mechanobiological mimicry of red blood cells to extend circulation times of hydrogel microparticles. Proc. Natl Acad. Sci. USA 108, 586-591 (2011).

31. De Angelis, A. A. \& Opella, S. J. Bicelle samples for solid-state NMR of membrane proteins. Nat. Protoc. 10, 2332-2338 (2007).

32. Muro, S., Garnacho, C. \& Champion, J. A. et al. Control of endothelial targeting and intracellular delivery of therapeutic enzymes by modulating the size and shape of ICAM-1-targeted carriers. Mol. Ther. 16, 1450-1458 (2008).

33. Mohanty, P., Lee, J., Glover, K. J. \& Landskron, K. Discoid bicelles as efficient templates for pillared lamellar periodic mesoporous silicas at $\mathrm{pH} 7$ and ultrafast reaction times. Nanoscale Res. Lett. 6, 61 (2011).

34. Weaver, J. C. \& Chizmadzhev, Y. A. Theory of electroporation: a review. Bioelectrochem. Bioenerg. 41, 135-160 (1996).

35. Glover, K. J. et al. Structural evaluation of phospholipid bicelles for solutionstate studies of membrane-associated biomolecules. Biophys. J. 81, 2163-2171 (2001).

36. Ma, Q. \& Wooley, K. L. The preparation of tbutyl acrylate, methyl acrylate, and styrene block copolymers by atom transfer radical polymerization: precursors to amphiphilic and hydrophilic block copolymers and conversion to complex nanostructured materials. J. Polym. Sci. A Polym. Chem. 38, 4805-4820 (2000).

37. Wang, X., Chen, J., Hong, K. \& Mays, J. W. Well-defined polyisoprene- $b$ poly(acrylic acid)/polystyrene- $b$-polyisoprene- $b$-poly(acrylic acid) block copolymers: synthesis and their self-assembled hierarchical structures in aqueous media. ACS Macro Lett. 1, 743-747 (2012).

38. Uhrig, D. \& Mays, J. W. Experimental techniques in high-vacuum anionic polymerization. J. Polym. Sci. A Polym. Chem. 43, 6179-6222 (2005).

39. Ma, Q. G. \& Wooley, K. L. The preparation of $t$-butyl acrylate, methyl acrylate and styrene block copolymers by atom transfer radical polymerization: precursors to amphiphilic and hydrophilic block copolymers and conversion to complex nanostructured materials. J. Polym. Sci. A Polym. Chem. 38, 4805-4820 (2000).

\section{Acknowledgements}

This material is based upon work supported by the National Science Foundation under Grants DMR-0906815 (D.J.P. and K.L.W.) and DMR-1105304 (K.L.W.) and the W.T. Doherty-Welch Chair in Chemistry, Grant No. A-0001 (K.L.W.). A portion of work was supported by the Materials Science and Engineering Division, US Department of Energy (DoE), Office of Basic Energy Sciences (BES) under Contract No. DEAC05-00OR22725 We thank the Keck Electron Microscopy lab at University of Delaware, Professor Chaoying $\mathrm{Ni}$ and Mr Frank Kriss for electron microscopy assistance. We thank $\mathrm{Mr}$ Sameer Sathaye, Ms Yingchao Chen and Mr Ngoc Nguyen for the atomic force microscopy assistance.

\section{Author contributions}

J.Z. and D.J.P. conceived the experiments and co-wrote the paper. J.Z. designed and carried out the experiments, collected and analysed the data. S.Z., K.Z., X.W., J.W.M. and K.L.W. provided the materials. All authors discussed the results and commented on the manuscript.

\section{Additional information}

Supplementary Information accompanies this paper at http://www.nature.com/ naturecommunications

Competing financial interests: The authors declare no competing financial interests.

Reprints and permission information is available online at http://npg.nature.com/ reprintsandpermissions/

How to cite this article: Zhu, J. et al. Disk-cylinder and disk-sphere nanoparticles via a block copolymer blend solution construction. Nat. Commun. 4:2297 doi: 10.1038/ncomms3297 (2013). 